\title{
Vehicle Photoelectric hybrid rotary System
}

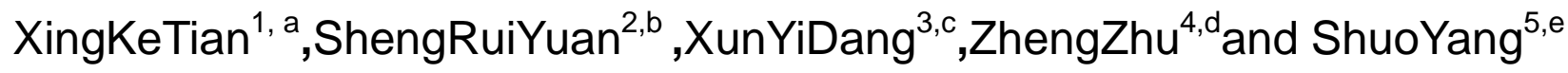 \\ ${ }^{1}$ China North Vehicle Research Institute, China \\ ${ }^{2}$ China North Vehicle Research Institute, China \\ ${ }^{3}$ China North Vehicle Research Institute, China \\ ${ }^{4}$ China North Industries Crop, China \\ ${ }^{5}$ China North Vehicle Research Institute, China \\ Atxk6305@sina.com, ${ }^{b}$ yuanshengrui@163.com, ${ }^{c} 12244270 @ q q . c o m,{ }^{d}$ zhuzh@norinco.cn, \\ eshujiang1412@126.com
}

\begin{abstract}
Keywords: Rotary platform, Optical communication, Photoelectric hybrid rotary connectors, optical transceiver.

Abstract.The current vehicle rotating platform uses the electric slip ring to realize the communication and power supply function between the platform equipment and the chassis equipment. With High-definition video, radar and other equipment installed in the vehicle system, on-board rotating platform to transmit large-capacity data and power supply the ability to put forward higher requirements. This paper presents a solution to realize the communication and power supply of the rotary platform by using the optical transceiver and the photoelectric hybrid rotary connector.
\end{abstract}

\section{Introduction}

In the traditional sense of vehicle-mounted weaponry, because of the electronic, digital level is not high, the internal transmission is the electrical control signal, rather than the digitized data information. However, with the development of electronic technology and the continuous improvement of digital level, automation and Intelligent have become the trend and necessary requirements.

And many of the Vehicle's weapons are equipped with a rotating body, such as tank turret, air defense anti-missile weapon system, unmanned combat remote control platform, reconnaissance vehicle rotating reconnaissance platform, etc., in the process of digitalization, these rotating platforms are equipped with a large number of radar, Infrared Thermal Imager, TV camera, laser rangefinder and other electronic equipment, Its data transmission is characterized by rotational connection, large capacity, high speed, and strong real-time performance[1]. These data are primarily required to be transferred to a central computer or an explicit control terminal in a tank chassis or a reconnaissance vehicle chassis.

\section{The present situation of the vehicle rotating platform and existing problems}

In the Vehicle system, the rotating platform is located on the top of the vehicle. The current vehicle rotating platform uses the electric slip ring to realize the communication and the power supply function between the platform equipment and the chassis equipment, but with the high-definition video, radar and so on a series of equipment installs to the vehicle system, the 
information quantity is very big and needs to rotate the transmission, receives the electrical characteristic, the material and the , it is imperative to improve the capacity of the vehicle rotating platform to transmit large capacity data and power supply by using the traditional electric slip ring to meet the demand.

The electric slip rings used on existing vehicles are almost entirely contact-type, its basic working principle is to slip through the brush between the two parts of the rotation of the signal connection, in the brush sliding process, will produce sparks, hundreds of amps large current of electromagnetic interference, brush contact resistance in the sliding process will change, These will seriously affect the reliability of high-speed data signal transmission, so the existing electric slip-ring solution only adapts to the transmission platform power supply, low-speed or DC control signal.

With the improvement of the digitalization of the vehicle, all kinds of high-speed digital signals need to be transmitted by the Rotary connectors, and the original electric slip rings are the "bottleneck" in the high-speed digital signal transmission[2]. At the same time, because of the need to transfer a large number of high-speed data between the top and the bottom, the vehicle internal communication network has very special requirements for data transmission, the performance of the data transmission such as speed, real-time, accuracy, fault-tolerant and reliability determines the overall performance of the electronic integrated system.

\section{The solution to the existing problem of the vehicle rotating platform}

Aiming at the requirement of large amount of communication data and high rate of the Rotary platform, this paper proposes to solve the data communication problem of the Rotary platform by using optical fiber communication of the combined photoelectric hybrid rotary connector.

The main features of optical fiber transmission technology are: (1) The bandwidth of fiber is very wide. The theory is up to 3 billion $\mathrm{MHz}$, can transmit image, voice and digital signal simultaneously; (2) Anti-jamming performance is good, electromagnetic interference, this is on-board system to apply optical communication is one of the important reasons; (3) Good confidentiality; (4) light weight, small size, Easy to install, (5) optical fiber communication is not charged, the use of safety, can be used for flammable, easy to violent sites, (6) The use of ambient temperature range.

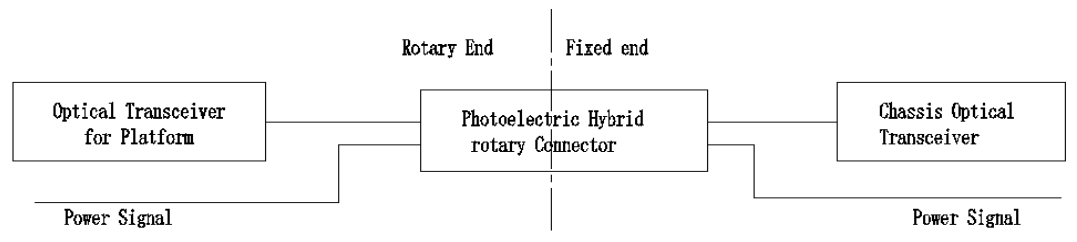

Fig.1 Configuration of the optical fiber communication system

The optical fiber communication system consists of a platform optical transceiver, a photoelectric hybrid rotary connector, a chassis optical transceiver three main parts, and the three are connected with 2 cables. The Platform optical transceiver and the chassis optical transceiver mainly realize multiplexing of video, audio, bus signal, serial communication signal and digital signal, encode and decode, and electro-optic/photoelectric conversion of signal. The Photoelectric hybrid rotary connector consists of an electric slip ring and a fiber optic rotary connector, Realize the rotating transmission of electric power and the bidirectional rotating transmission of large capacity optical signal. 


\section{The design and realization of the vehicle rotating platform}

\section{System Structure}

The vehicle rotation platform based on a project needs to realize 3-channel HD CCD, 3-channel PAL video, 1-way audio two-way communication, 2-way RS422, 1-channel can bus, 7-channel digital rotary transmission, in addition, the need to provide 10-channel signal transmission electric slip ring (which 5-way input, 5 output), 5-channel high-power transmission electric slip ring (2 50A power transmission electric slip Ring, 3 10A power transmission electric slip Ring), 2-channel DC 270V High voltage power transmission slip ring.

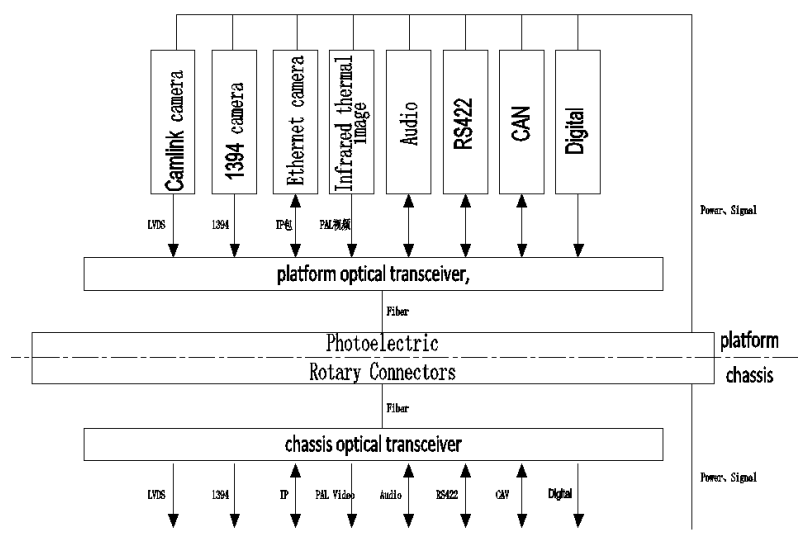

Fig.2 System diagram

The system diagram is shown in the diagram. The whole optical fiber communication system is composed of three main parts, such as platform optical transceiver, photoelectric hybrid rotary connector and chassis optical transceiver. Platform Optical transceiver and chassis optical transceiver mainly achieve video, audio, bus signals, serial communication signals and digital signal multiplexing, encoding and decoding, as well as the signal of the electro-optic / photoelectric conversion; The photoelectric hybrid rotary connector consists of an electric slip ring and a fiber optic rotary connector, which realizes the rotating transmission of electric power and the bidirectional rotation of the optical signal.

\section{Choice of fiber}

As the optical fiber communication system is applied to the vehicle platform, optical fiber connection length is short, without considering the effect of dispersion, and the optical path through the wall is more frequent, so the loss characteristics caused by the signal damage than the dispersion characteristics of the signal deformation of the system, the more the multimode fiber is more suitable for the application of the system conditions. Moreover, compared with single-mode fiber, the fiber core is thicker, the collimator alignment of the fibre-optic rotary connector is easier, and the insertion loss can be reduced, so we choose multimode fiber.

\section{Optical Transceiver}

The equipment of realizing the optical fiber conversion of the two ends of the vehicle rotating platform system we call it the optical transceiver. Optical transceiver is a transmission device in optical fiber communication, which carries out photoelectric conversion and transmission. This system uses digital optical transceiver, digital optical transceiver will be transmitted to the image, voice and data signals to digital processing, and then the digital signal multiplexing, so that the Low-speed digital signal conversion to the high speed signal, and the signal into a light signal. At the receiving end, the optical signal is reduced to an electrical signal, the restored high speed signal decomposes the original low-speed signal, and finally the data signal is restored to the image, speech and data signal. 
The digital optical transceiver used in this system is mainly composed of optical transmitter, optical fiber and optical receiver. The basic composition is shown in Figure 3. In practical applications, both sides of optical fiber often need simultaneous communication, that is, both optical transmitter and optical receiver at both ends. The development of technology has made the optical transmitter and optical receiver integrated into a module, referred to as the optical module.

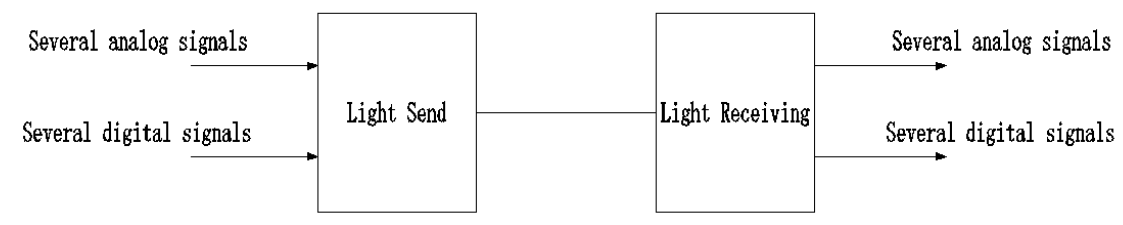

Fig.3 The composition of digital optical transceiver

The optical transceiver of the system includes a platform optical transceiver and a chassis optical transceiver, since we choose to use a single channel fiber-optic rotary machine to complete the data transfer function of the Rotary platform, and by using CWDM to extend the transmission capability of a single channel fiber optic rotary connector, we consider that we choose multimode fiber, which uses a large transmitting light power, The receiver has a better sensitivity to the optical module.

PAL video signal, audio signal, RS422 signal, can bus signal and other signals are relatively low speed signals, the code is multiplexed into a way optical signal transmission, using $1.25 \mathrm{G}$ optical modules can meet the requirements; Gigabit Ethernet, Camera link, and 1394 interfaces are all high speed signals, with gigabit Ethernet needing 1. 25G Optical module matching, Camera link interface in base mode maximum rate of $2.5 \mathrm{Gbps}$, but the effective data transmission of the camera used by the Rotary platform is 10 bits, the pixel clock is $50 \mathrm{M}$, and used $1.25 \mathrm{G}$ Optical module is sufficient to meet the requirements, 1394 signal is also used 1. 25G Optical module Matching, we select the 8-channel CWDM with single channel fiber-optic rotary connectors to achieve 8-way data transmission. The Wavelength division multiplexing apparatus is used in the index of table 1.

table 1 Technical specifications of 8-channel wavelength Division multiplexer

\begin{tabular}{|l|l|l|}
\hline Parameter name & Index value & per unit \\
\hline Fiber type & $62.5 / 125$ & um \\
\hline Fiber length & 1 & $\mathrm{~m}$ \\
\hline Connection mode & $\mathrm{SC}$ & \\
\hline Working temperature & $-40 \sim 55$ & ${ }^{\circ} \mathrm{C}$ \\
\hline Storage temperature & $-55 \sim 85$ & ${ }^{\circ} \mathrm{C}$ \\
\hline IL Temp Drift & $\leq 0.006$ & $\mathrm{Db} /{ }^{\circ} \mathrm{C}$ \\
\hline Max Power Handling & 300 & $\mathrm{~mW}$ \\
\hline Channel interval & 20 & $\mathrm{~nm}$ \\
\hline Working bandwidth & Center wavelength \pm 6.5 & $\mathrm{~nm}$ \\
\hline Number of channels & 8 & \\
\hline Center Wavelength & 1471149115111531 & $\mathrm{~nm}$ \\
\hline Adjacent Isolation & 1551157115911611 & \\
\hline echo Loss & $\geq 30$ & $\mathrm{~dB}$ \\
\hline
\end{tabular}

After using WDM technology, the performance of the photoelectric hybrid rotary connectors has not decreased and the number and capacity of the transmitted channels are significantly increased, so it is successful to extend the transmission capacity of single channel rotary connectors with 


\section{WDM technology[3].}

\section{Photoelectric hybrid rotary connectors}

The electric slip ring is the earliest rotary connector, and the electric slip ring transmits electric currents and data signals from the rotating metal ring to the stationary ring, depending on the brush and the ring wall. In a rotating connection system, the electric slip ring transmits low bandwidth digital or analog signals and DC or AC power currents from a stationary platform to a rotating platform, or transmits digital or analog signals and DC or AC power currents from a rotating platform to a stationary platform. The electric slip ring guarantees the continuous transmission of the signal and the current, is the connection medium between the stationary platform and the rotating platform, the traditional rotary connector is realized by the electric slip ring, in this system, we use the traditional electric slip ring to realize the power transmission.

A fiber optic rotary connector is similar to an electric slip ring, which is composed of a rotor (a rotating platform) and a stator (stationary platform). The optical signal is transmitted from the rotor through the rotating surface and then from the stator part; The purpose of a fiber optic rotary connector is to minimize the loss of light and rotate the amount of the light signal through the rotating plane. The fiber optic rotary connector is divided into a multi-channel fiber optic rotary connector and a single channel fiber optic rotary connector, depending on the number of glazing channels of the rotary connector. Multi-channel fiber optic rotary connector structure is more complex, in addition to the need for optical lenses also need some mechanical support mechanisms, such as gears, magnetic mechanisms and so on. So in this system, we choose the wavelength division multiplexer combination single channel rotary connector to achieve optical fiber communication, low cost, simple structure.

In this system, the whole structure of the photoelectric hybrid rotary connector is composed of three layers inside and outside, and the outer layer is the electric slip ring combination of the multi-channel transmission power, the middle layer is the electric slip ring combination of the electrical signal transmission, the most inner is the optical fiber rotary connector in the middle of the transmission signal electric. The specific indices are as follows.

(1)Fiber optic Rotary connectors

I Channel: 1 optical fiber

I Data transfer rate $\geq 2 \mathrm{GBo}$

I Insertion Loss $\leq 3 \mathrm{db}$

I Insertion Loss Typically Changes $\leq 0.5 \mathrm{db}$

I Crosstalk $\geq 40 \mathrm{db}$

I Rotational Speed $\leq 120 \mathrm{rpm}$

I Working temperature: $-40^{\circ} \mathrm{C} \sim+55^{\circ} \mathrm{C}$

(2)Electrical slip ring

I signal transmission electric slip ring :10 (5-way input, 5 output)

I high-power transmission electric slip ring: 5 (2 50A power transmission , 3 10A power transmission)

I DC 270V High voltage power transmission :2

I The maximum friction force $\leq 20 \mathrm{NM}$

I Working temperature: $-40^{\circ} \mathrm{C} \sim+55^{\circ} \mathrm{C}$

I Speed $\geq 60 \mathrm{t} / \mathrm{min}$

video, audio, digital, RS422, can bus optical communication implementation

The system involves video, audio, RS422, can bus, digital data signal transmission, because most of the signal transmission rate is low, here using FPGA to achieve low speed signal synchronization, 
multiplexing/demultiplexing. The principle of optic-audio card optical fiber communication is shown in Figure 4.

(1) Video channel

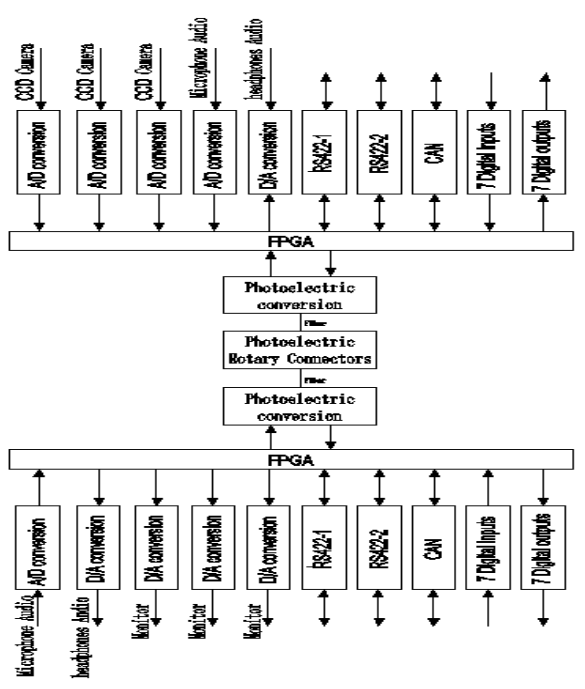

Fig.4 Video optical diagram

The video transmission module is composed of a camera or other output video signal through video amplification, low-pass filtering, clamp, high-speed A/D transform several functional blocks. The video amplification chip provides the high performance video signal filtering shaping processing, the ad chip transforms the video amplifier processing the analog signal to the digital signal. The video receiving module converts the digital signal used by FPGA to D/A, then passes the Low-pass filter, then outputs the analog video signal after power amplification, as shown in Figure 5.

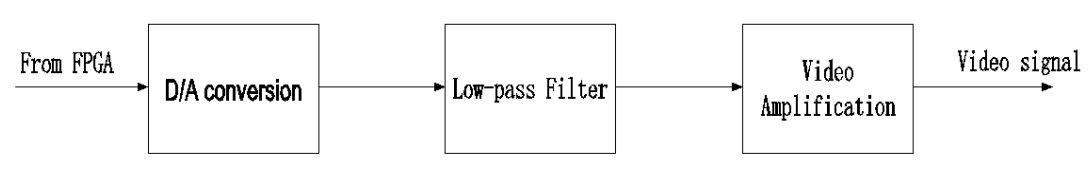

(2) Audio channel

Fig.5 Schematic diagram of video channels

The audio transmitting module transfers the audio signal from the mike to the FPGA after the audio is filtered, $\mathrm{A} / \mathrm{D}$ conversion, and the audio receiving module converts the digital signal used by FPGA to D/A, then outputs the audio signal by amplifying the audio.

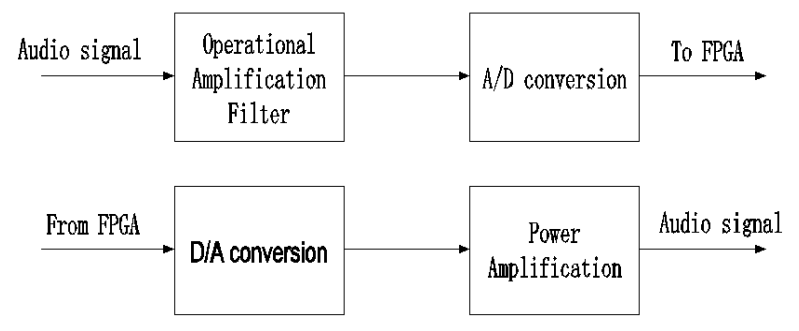

Fig.6 Schematic diagram of audio channels

(3) RS422 Channel

Because RS422 does not need A/D conversion, only a certain isolation means to connect with the FPGA, the differential input needs to add 120 Euclidean matching resistance. 

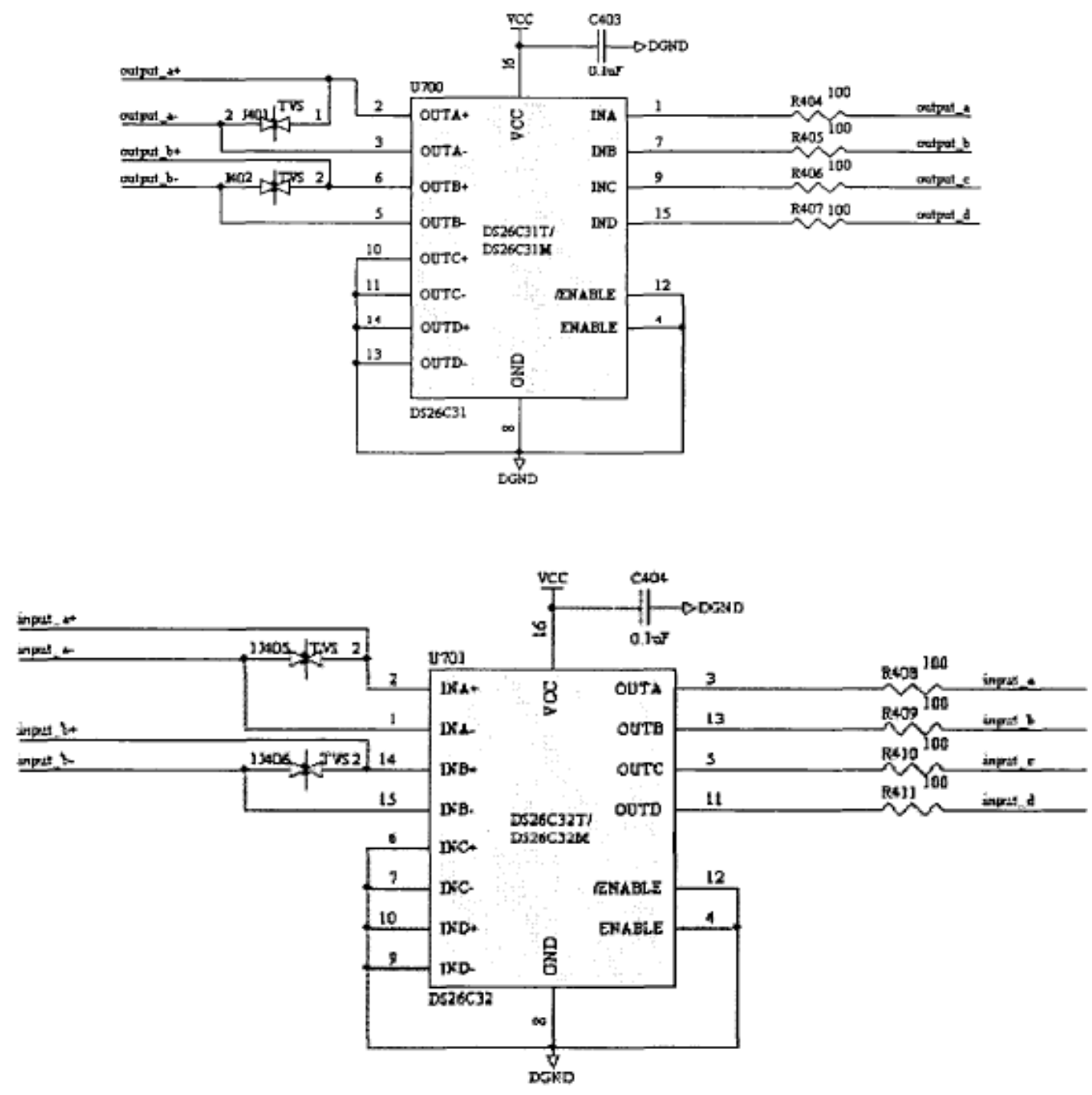

(4) Digital quantity

Fig.7 RS422 Transceiver circuits

7-Channel digital IO access FPGA before using ps2805-4 to the input photoelectric isolation. For $24 \mathrm{~V}$ input, use a partial voltage resistor.
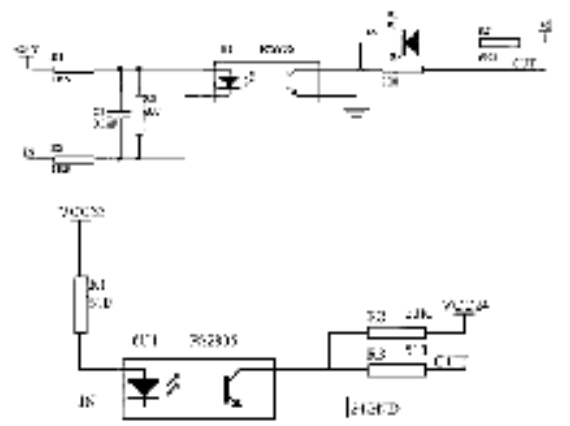

Fig.8 Digital isolation circuit

(5) Can bus

The CAN bus transceiver is used to realize the data exchange and voltage isolation between the can bus and FPGA, and the driving ability is increased. Can bus differential output terminal needs to receive 120-Euclidean matching resistance. 


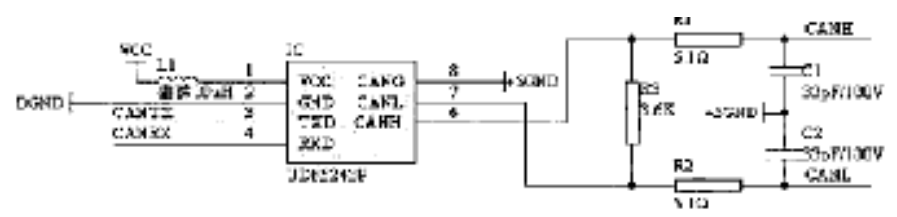

Fig.9 CAN circuit

\section{Gigabit Ethernet Optical Transmission}

Over $80 \%$ of the existing LAN in our country is Ethernet. Ethernet has the advantages of low price, high reliability, good scalability and easy management, and now it is widely used in $10 \mathrm{M}$ Ethernet and 100M bit Fast Ethernet two versions. Dry Gigabit Ethernet supports switches to switches, switches to terminals, new Full-duplex operating modes for use, and Half-duplex operating modes for shared connections. It uses a fiber, unshielded twisted pair (CATS) or coaxial cable as the transmission medium.

Gigabit Ethernet is just the technology of the data link layer and the physical layer. It does not require any changes to the application of higher-level protocols. Existing applications can operate on an Ethernet without distinction, regardless of the rate at which the $10 \mathrm{mb} / \mathrm{s}$ is used, $100 \mathrm{mb} / \mathrm{s}$ or $1000 \mathrm{mb} / \mathrm{s}$.

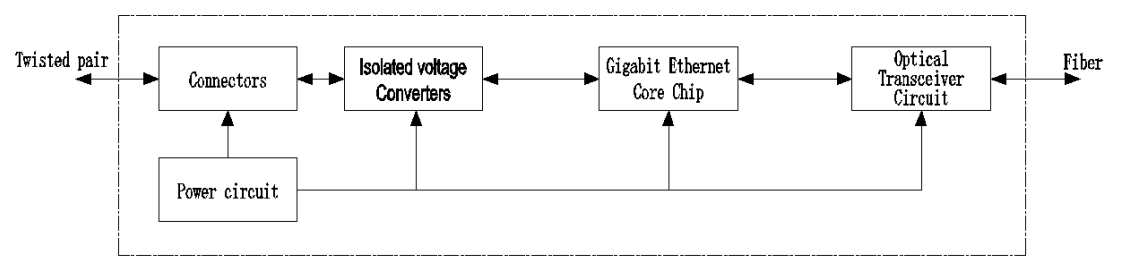

Fig.10 Ethernet fiber optic transmission

Gigabit Ethernet Core Chip is the core of the design, the selection is 88e1111s. According to the function of the design target and the requirement of $88 \mathrm{e} 1111 \mathrm{~s}$ to the periphery circuit, the related pin periphery circuit is determined. 


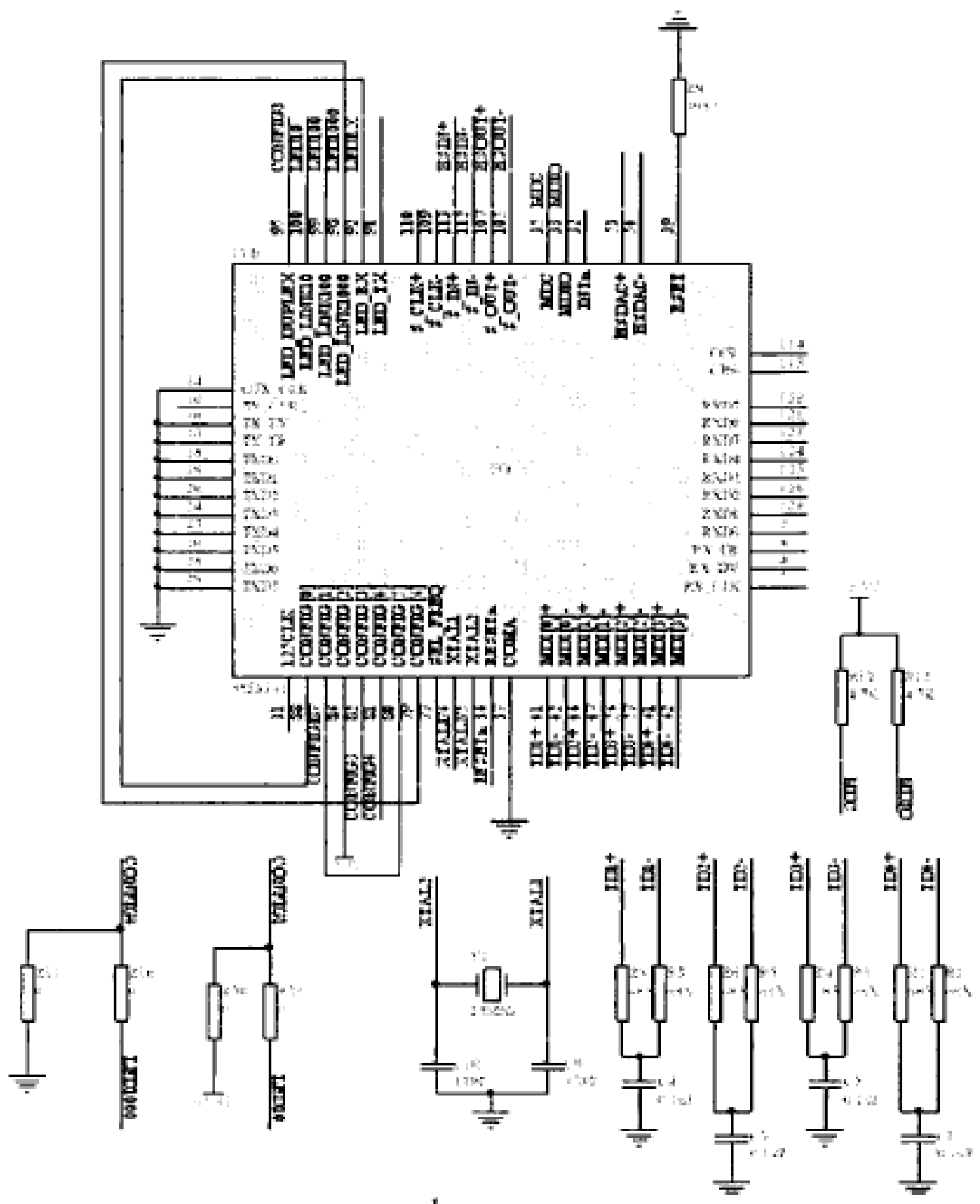

Fig.11 Optical core circuit

Gigabit Ethernet Optical Transceiver circuit using optical module, select High-performance Optical module, support 1. 25Gbps transmission rate, duplex dual-fiber transceiver, with duplex SC fiber interface and PECL electrical interface, which is defined as table 2. 1-5 of the pins are used for the receiving end, and 6-9 pins are used for the transmitter end, of which 4 feet are signal detection (SD) PIN, when the fiber link is normal, the SD output logic is high, while the fiber link is not normal, SD is low logic.

table 2 Optical module PIN definition

\begin{tabular}{|l|l|l|}
\hline PIN & Signal & Description \\
\hline 1 & RX GND & Receiving data GND \\
\hline 2 & RD + & Receive data output + \\
\hline
\end{tabular}




\begin{tabular}{|l|l|l|}
\hline 3 & RD- & Receive data output - \\
\hline 4 & SD & Signal detection output \\
\hline 5 & RX VCC & Receiving power \\
\hline 6 & TX VCC & Send Power \\
\hline 7 & TD- & Launch data Input- \\
\hline 8 & TD + & Launch data Input + \\
\hline 9 & TX GND & Launch data GND \\
\hline
\end{tabular}

Fig. 12 is part of the isolating transformer. The isolating transformer mainly has the signal transmission, the impedance match, the waveform Repair, signal clutter suppression and high voltage isolation and other functions.

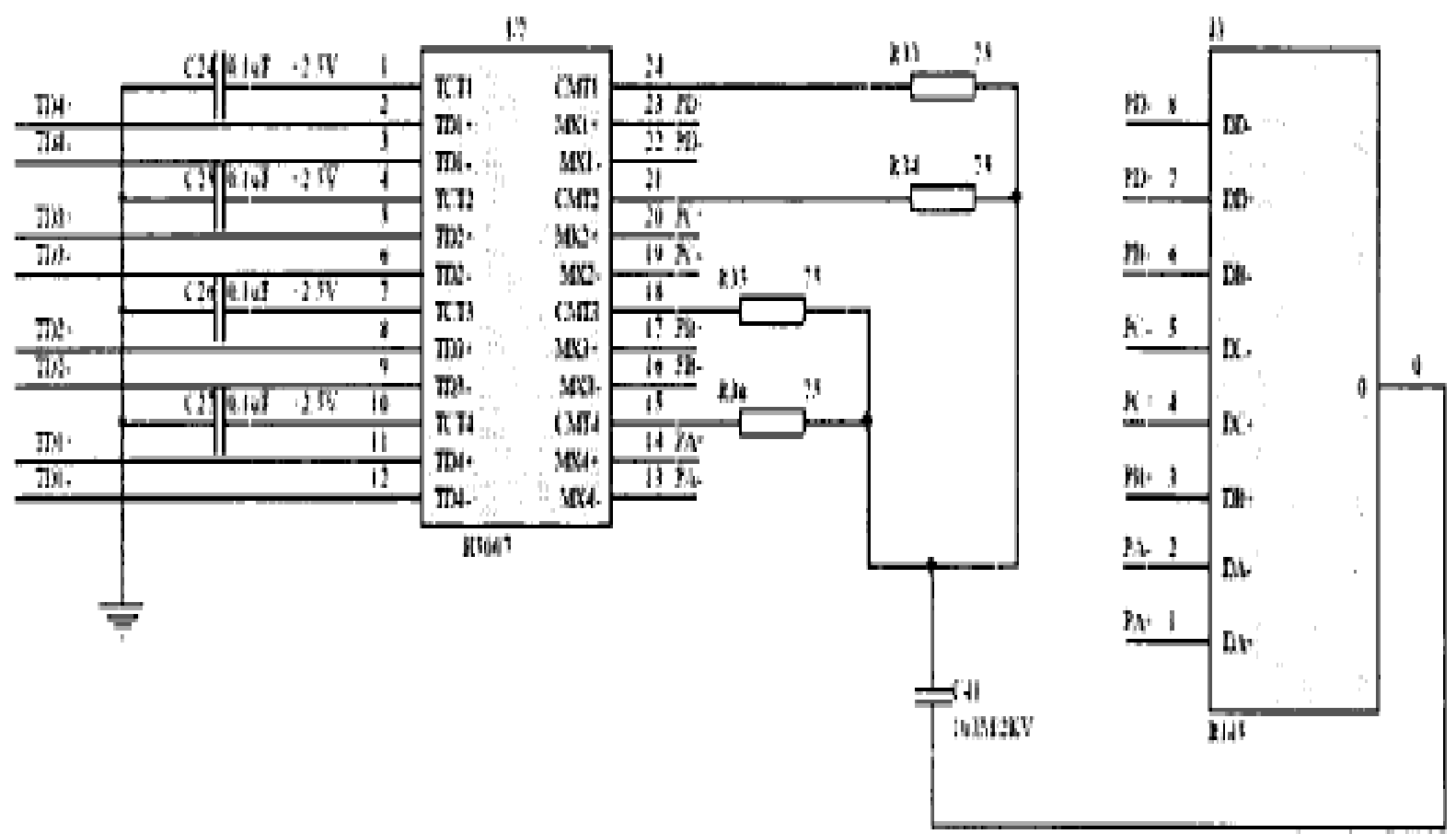

Fig.12 isolation transformer circuit

In this system, the isolation transformer mainly has three kinds of uses, one, enhances the signal, make the transmission distance farther; second, the chip end and external isolation, anti-interference ability greatly enhanced, and the chip added a great deal of protection; third, when receiving a different level (if some chip is $2.5 \mathrm{~V}$, some chips are $3.3 \mathrm{~V}$ ) Network, it will not affect each other's devices.

\section{The significance and application foreground of the onboard photoelectric hybrid rotary platform}

The future war is the Informationization, the digitized war, in order to win the information war, dominate the battlefield maneuver right, the implementation accurate attack, provides the effective protection and the safeguard goal, the new weapon system will use the digitized command and control system, the electronic warfare equipment and the precision guidance weapon and so on. Multi-channel image information, voice, network, control information and rotary connection of the combat equipment, such as self-propelled artillery, tanks, armored vehicles, anti-aircraft missile turret and chassis; The battlefield situation sensing device, such as radar and laser tracker, which is equipped with rotary linkage, needs optical communication technology to transmit a large amount 
of data such as image information and network[4]. Therefore, it is one of the key of information integration to study the optical fiber communication of vehicle rotating platform, which has far-reaching significance for the development of weapon equipment in the near, middle and long term, and plays a key supporting role in the information technology of weapon equipment.

\section{References}

[1] DaGongJia, Development of multi-channel space interconnection optical rotary connectors[D].TianJin:TianJin University,2004.

[2] YongChen,HaiChengYang,WeiMingWang.The technology of photoelectric combination converge ring[J].Apply Optical rotation,2002,23(6):12-15.

[3] DaGongJia,WenCaiJing, YiMoZhang,JinZhang,FengTang.Design of low loss rotary connectors[J].Photon Technology,2005,9(3):138-139.

[4] YingQingQian,RunHuaXu,HuChenSun,JianPingZhou.Research on interconnection Technology of optical Module PECL interface[J].2009,28(5):1-2. 